\title{
Morphological variability of the trabecular meshwork in glaucoma patients: implications for non-perforating glaucoma surgery
}

\author{
Thomas S Dietlein, Philipp C Jacobi, Christoph Lüke, Günter K Krieglstein
}

\begin{abstract}
Backgroundlaims-Morphological variability of the trabecular meshwork could be of considerable importance for the proper intraoperative outcome of nonperforating antiglaucomatous surgery, such as deep sclerectomy and viscocanalostomy. The aim of this study was therefore to assess qualitative and quantitative characteristics of the trabecular meshwork in glaucoma patients undergoing trabeculectomy.
\end{abstract}

Methods-Trabeculectomy specimens from 177 glaucoma patients were prepared for light microscopy; 100 specimens were found to be suitable for qualitative assessment and quantitative computerised image analysis; measurements were taken of the meridional diameter of Schlemm's canal as well as the thickness of the trabecular meshwork at different positions.

Results-The mean meridional diameter of Schlemm's canal was $290 \mu \mathrm{m}$ with the smallest values in the young patients with infantile and secondary glaucomas. the thickness of the trabecular meshwork ranged between $50-70 \mu \mathrm{m}$ in the anterior region and between $100-130 \mu \mathrm{m}$ for the posterior portion. The thickness of the anterior meshwork significantly decreased with age. The pigmentation of excised trabecular meshwork was found to be weak or even lacking in 68 patients. In 20 glaucoma patients the uveal meshwork was covered by an endothelial layer.

Conclusions-From the morphological point of view the risk of inadvertent perforation during deep sclerectomy in older, white glaucoma patients should be taken into account even by an experienced surgeon, because the anterior meshwork in these cases is very thin and trabecular pigmentation that can be used as a

Accepted for publication 28 June 2000

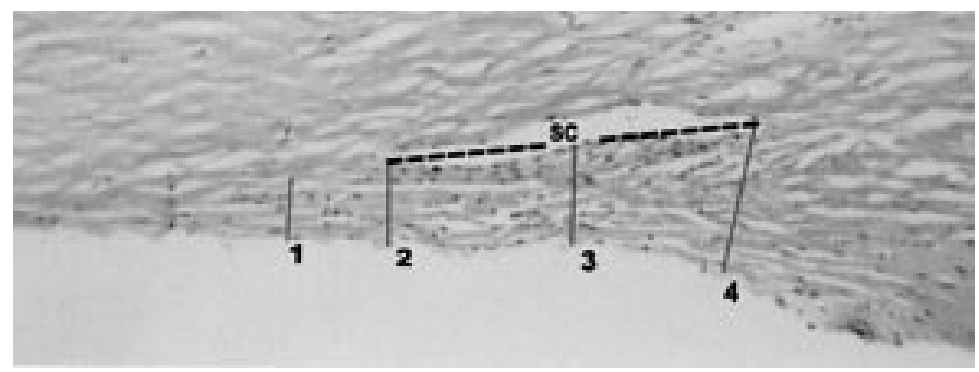

Figure 1 Trabecular meshwork in a 67 year old patient with primary open angle glaucoma revealing no pigmentation. The broken line shows the meridional diameter of Schlemm's canal (SC), lines 1-4 indicate the positions where the thickness of the trabecular meshwork was measured. Original magnification $\times 134$. topographic landmark is often lacking. The functional success of non-perforating glaucoma surgery in many patients may be limited by endothelial covering of the trabecular meshwork.

(Br f Ophthalmol 2000;84:1354-1359)

Many new non-perforating antiglaucomatous procedures have been introduced in recent years, including viscocanalostomy and deep sclerectomy with or without a device. ${ }^{1-5} \mathrm{~A}$ critical step in this technique is the dissection of a deep scleral flap, thereby deroofing Schlemm's canal and preserving the structural integrity of the trabecular meshwork. The conceptual advantages of this surgical strategy are the reduction of intraocular irritation, shortening of visual rehabilitation, and a lower risk profile than that associated with conventional filtering surgery. ${ }^{156}$ However, the surgical technique is extremely demanding and carries certain intraoperative risks. The most frequently cited intraoperative complication is the perforation of the trabeculo-descemetic membrane with an incidence of around $10 \% .^{58}$ Dissecting at too superficial a level or difficulty in finding Schlemm's canal and its ostia are further common problems. ${ }^{9}$

Most authors practising this form of surgery emphasise that adequate experience with the technique is essential to guarantee a satisfactory intraoperative outcome. ${ }^{5-9}$

In order to assess the influence of morphological variabilities on the intraoperative problems of deep sclerectomy we investigated qualitative characteristics and quantitative topography of the trabecular meshwork and Schlemm's canal in a large representative series of glaucoma patients who had undergone conventional trabeculectomy.

\section{Materials and methods}

Trabeculectomy specimens of 177 consecutive glaucoma patients were collected for light microscopy between 1996 and 1999. Trabeculectomy was performed by one surgeon using a standardised procedure. Vasoconstrictive agents (for example, ornipressin) were administered preoperatively. After placing a superior rectus muscle bridle suture a limbus based conjunctival flap was prepared in the upper circumference. Cauterisation was avoided if possible. A rectangular scleral flap of around half the scleral thickness and $4 \mathrm{~mm}$ long basis at the limbus was dissected using a crescent diamond knife. Two non-resorbable sutures (Nylon 10-0) for fixation of the scleral flap were brought into position without a knot. 


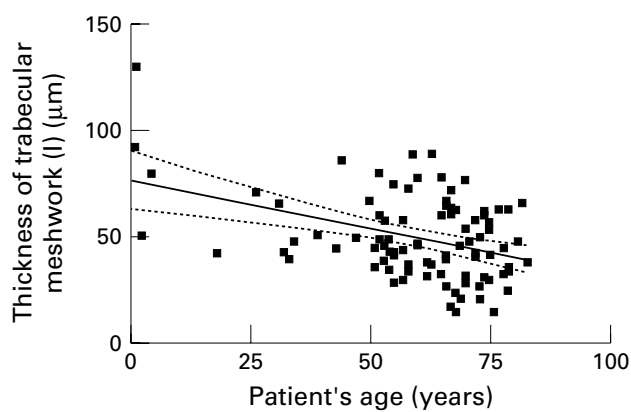

Figure 2 Linear regression line with 95\% CI describing the relation between age and thickness of the trabecular meshwork at position 1: slope is $-0.44(0.11) ; r^{2}=0.16$; $p<0.0001$.

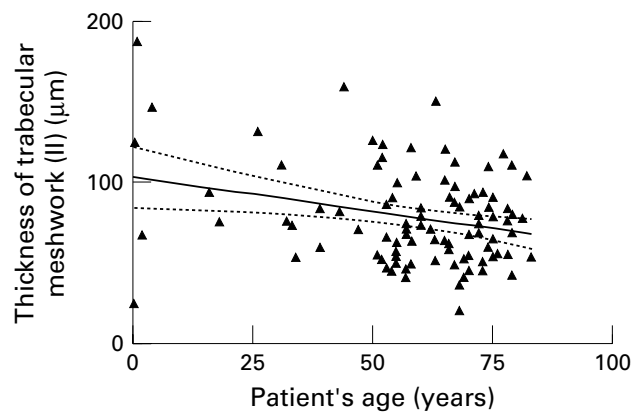

Figure 3 Linear regression line with 95\% CI describing the relation between age and thickness of the trabecular meshwork at position 2: slope is $-0.42(0.15) ; r^{2}=0.07$; $p=0.006$.

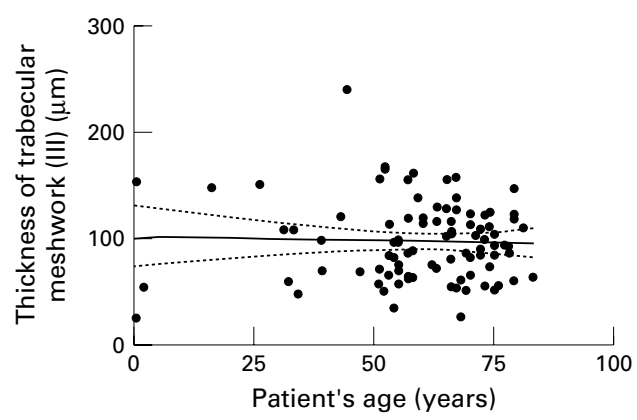

Figure 4 Linear regression line with 95\% CI describing the relation between age and thickness of the trabecular meshwork at position 3: slope is $-0.07(0.23) ; r^{2}=0.001$; $p=0.75$.

Thereafter, a $1.5 \times 3.0 \mathrm{~mm}$ sized trabeculectomy block was excised using a very sharp diamond knife and avoiding any distortion of the trabecular tissue by the forceps. After performing the peripheral iridectomy the scleral sutures were tied and the conjunctiva was closed with a running Vicryl 8-0 suture.

Trabeculectomy specimens were immediately placed into buffered formalin solution and fixed by immersion overnight. They were then dehydrated in graded alcohol and embedded in paraffin. The rectangular tissue block was cut as close to the meridional direction as possible. Relatively thick sections of $5 \mu \mathrm{m}$ were used to guarantee structural integrity. Serial sections were stained with haematoxylin and eosin and examined by light microscopy.

Only one trabeculectomy specimen from each patient was considered for statistical reasons. The following essential data were collected for each patient: (a) age, (b) glaucoma subtype, (c) duration and stage of the disease, (d) maximal recorded intraocular pressure according to patient's declaration, (e) refractive state of the eye, and ( $f$ ) history of previous intraocular surgery and laser trabeculoplasty.

Seventy seven of 177 trabeculectomy specimens were excluded from the study, because they showed marked morphological artefacts related to the surgical dissection or did not contain sufficient trabecular tissue with a satisfactory level of meshwork integrity for reliable topographic and qualitative assessment of the trabecular morphology. Sections from the edges of the specimens were generally discarded in order to exclude artefacts produced by the diamond knife or the fine forceps.

Photographs of the microscopic sections were taken and analysed using automated image analysis software (SigmaScan Pro, Microsoft, USA) at a magnification of between 500 and $1000 \times$. In the meridional sections, measurements were taken of the anteriorposterior diameter of Schlemm's canal and the thickness of the trabecular meshwork at the following positions: (1) $100 \mu \mathrm{m}$ anterior to the top of Schlemm's canal, (2) at the top of Schlemm's canal, (3), halfway down the canal, and (4) and at the end of the canal (5). The thickness of the trabecular meshwork was always measured perpendicular to the inner layer of the uveal meshwork (Fig 1). Measurements were taken on one randomly selected section of the central part of the tissue specimen. In order to assess fluctuations within the trabeculectomy specimens we compared the measurements of each variable in two different randomly selected sections of the same specimens in 10 patients. Qualitative assessment comprised the presence of intracanalicular tissue/septae, of tangential drainage vessels ("bridge-like channels"), of an endothelial cell layer covering the uveal trabecular meshwork and of any pigmentation of the trabecular tissue. Histological analysis was performed without knowledge of the patients' data.

All quantitative data were evaluated using statistical software (Prism 2.0, GraphPad, USA). Statistical analysis comprised linear regression and the Mann-Whitney and Wilcoxon rank tests. For comparison of reproduc-

Table 1 Mean thickness (SD) of the trabecular meshwork ( $\mu$ m)

\begin{tabular}{|c|c|c|c|c|}
\hline & $I$ & $I I$ & III & $I V$ \\
\hline POAG $(n=41)$ & $48(17)$ & $78(25)$ & $103(32)$ & $132(48)$ \\
\hline PEX-Gl $(n=30)$ & 47 (19) & $71(21)$ & $92(28)$ & $121(38)$ \\
\hline Pigm Gl $(n=11)$ & $43(9)$ & $68(22)$ & $80(32)$ & $104(32)$ \\
\hline Infant $\mathrm{Gl}(\mathrm{n}=9)$ & $78(31)$ & $107(51)$ & $107(61)$ & $143(98)$ \\
\hline $\operatorname{Sec} \mathrm{Gl}(\mathrm{n}=9)$ & $58(20)$ & $89(42)$ & $106(58)$ & $143(76)$ \\
\hline Total $(\mathrm{n}=100)[\min -\max ]$ & $50(20)[15-130]$ & $78(29)[21-188]$ & $97(36)[26-240]$ & $126(51)[33-274]$ \\
\hline
\end{tabular}




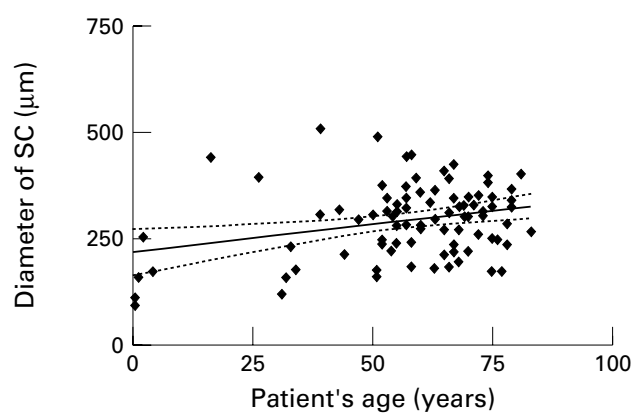

Figure 5 Linear regression line with 95\% CI describing the relation between age and meridional diameter of Schlemm's canal: slope is $1.3(0.44) ; r^{2}=0.09 ; p=0.004$.

ibility in 10 specimens we used a paired, two tailed $t$ test. Significance level was $\mathrm{p}<0.05$.

\section{Results}

PATIENTS

The mean age of our 100 patients was 57.7 (SD 19.7) years (range, minimum 4 months and maximum 83 years). Clinical diagnosis was primary open angle (POAG) $(n=41$, mean age 64 years), exfoliative (PEX-Gl) $(n=30,69$ years), pigment dispersion (Pigm Gl) $(n=11$, 55 years), infantile (Infant Gl) ( $\mathrm{n}=9,8$ years), and secondary glaucoma (Sec Gl) $(n=9,46$ years). Secondary glaucomas included eyes with uveitis $(n=3)$, post-traumatic glaucomas $(n=3)$ as well as glaucomas caused by an iridocorneo-endothelial (ICE) syndrome $(n=2)$ or

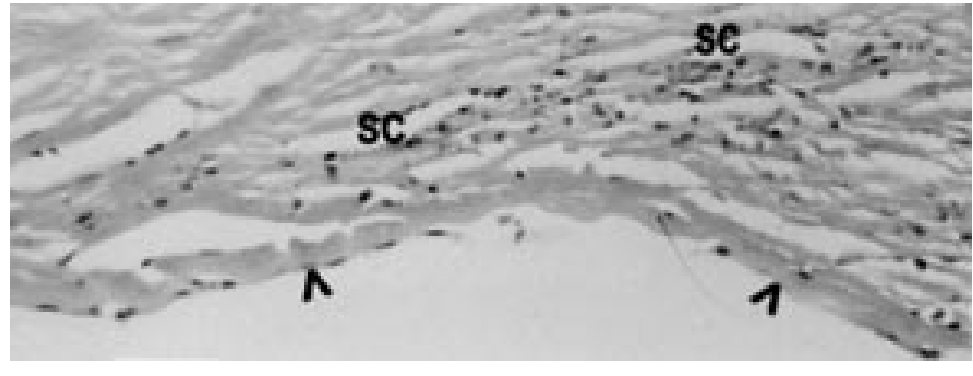

Figure 6 Trabecular meshwork in a 57 year old patient with irido-corneo-endothelial (ICE) syndrome and secondary glaucoma. The lumen of Schlemm's canal (SC) is bridged by several tissue bands. The uveal meshwork is completely covered by a thick endothelial layer (arrowheads). Original magnification $\times 309$.

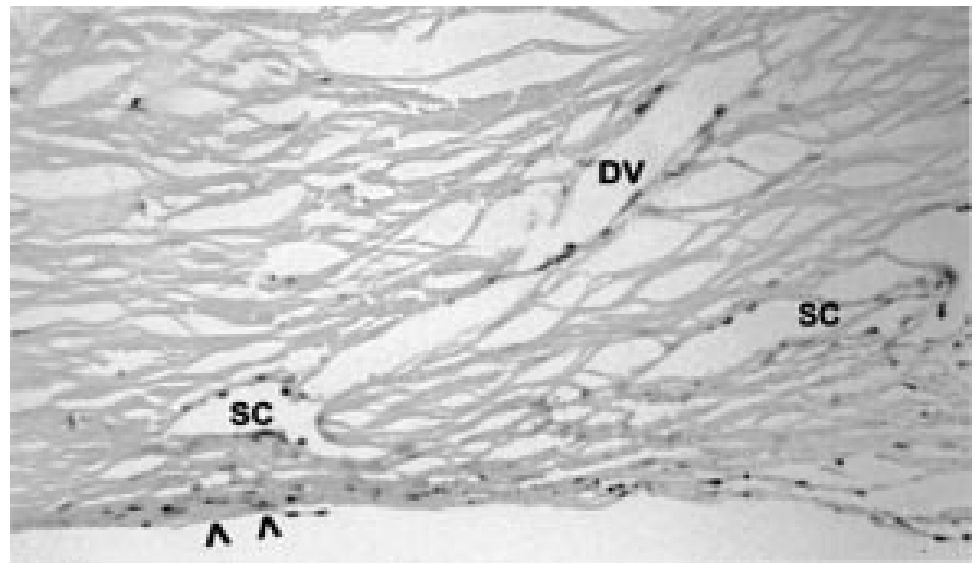

Figure 7 Trabecular meshwork in a 72 year old patient with primary open angle glaucoma and history of laser trabeculoplasty. Schlemm's canal (SC) consists of separated lumina; a radially running drainage vessel (DV) meets the anterior part of Schlemm's canal. The anterior meshwork shows a loss of intertrabecular space and an endothelial covering (arrows). Original magnification $\times 244$. by Rieger's anomaly $(n=1) ; 96$ patients were white, four patients came from the Middle East.

Previous intraocular surgery had been reported in 27 eyes including filtering surgery in 16 eyes, cataract surgery in six eyes, and multiple surgical interventions in five eyes. A history of laser trabeculoplasty was documented in 48 eyes.

Mean maximum intraocular pressure was 38.7 (SD 9.8) $\mathrm{mm} \mathrm{Hg}$ (range $24-80 \mathrm{~mm} \mathrm{Hg}$ ), mean duration of glaucoma history was 10.3 (8.1) years (range 0.1-36 years). Mean ocular refraction was -1.5 (4) dioptres (range -15 dioptres to +6 dioptres).

QUANTITATIVE ASSESSMENT OF THE

TRABECULECTOMY SPECIMEN

The values obtained for the thickness of the trabecular meshwork showed a Gaussian normal distribution. Although range of values was wide (Table 1), thicknesses of the anterior meshwork $(1+2)$ could be seen to decrease significantly with age, while the values for the central and posterior meshwork $(3+4)$ did not (Figs 2-4 ). Comparing patients with primary open angle glaucoma, exfoliative and pigment glaucoma with the young patients with infantile and secondary glaucomas, the differences in thickness of the anterior trabecular meshwork between old and young were significant, whereas no age difference was observed for the thickness of the central and posterior meshwork. No significant correlation was found between the thickness of the meshwork and the duration of glaucoma history, maximum intraocular pressure, ocular refraction, or previous intraocular surgery.

The mean anterior posterior diameter of Schlemm's canal was 290.8 (84) $\mu$ m (range 92-508 $\mu \mathrm{m}$ ). This diameter significantly increased with age and displayed the smallest values in the young patients with infantile and secondary glaucomas (Fig 5). No correlation was found between the size of Schlemm's canal and the duration of glaucoma history, maximum intraocular pressure, ocular refraction, or previous intraocular surgery.

Statistical comparison of two randomly selected quantitative data sets of 10 trabeculectomy specimens revealed a correlation coefficient between 0.88 and 0.96 for the trabecular thickness and meridional size of Schlemm's canal.

QUALITATIVE ASSESSMENT OF THE

TRABECULECTOMY SPECIMEN

The uveal trabecular meshwork was completely covered by an endothelial layer in 10 patients (Fig 6) and partially covered in another 10 patients (Fig 7). Among these 20 patients five had secondary glaucomas, two had infantile glaucoma, and 13 had open angle glaucomas. Out of these 13 adult patients 11 had a history of previous laser trabeculoplasty.

Identification of Schlemm's canal was feasible in all patients by different magnifications of light microscopy. A bifid canal lumen was seen in only three patients (Fig 7). Tissue bands bridging Schlemm's canal or fine intracanal- 


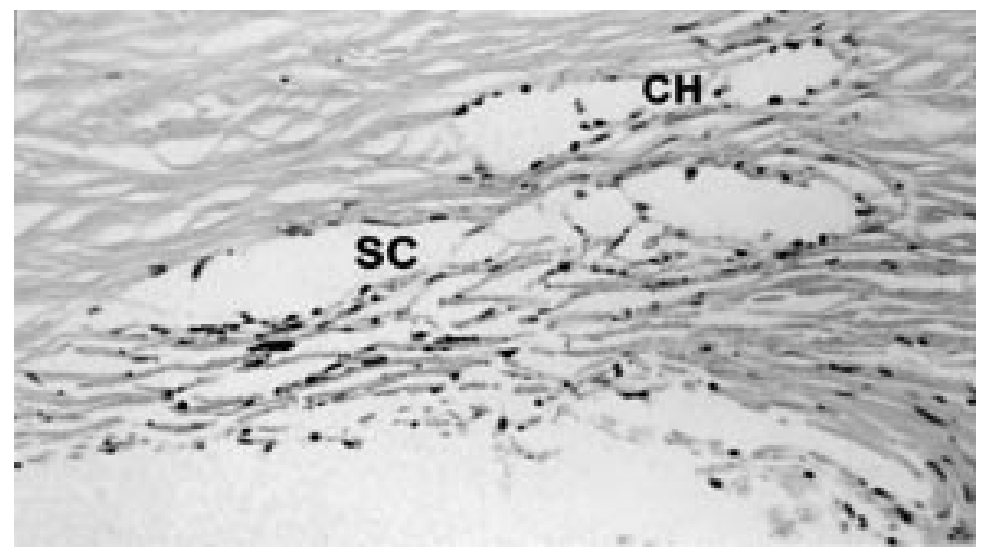

Figure 8 Trabecular meshwork in a 65 year old patient with exfoliative glaucoma showing moderate pigmentation. A "bridge-like channel" $(\mathrm{CH})$ goes tangentially along with Schlemm's canal (SC) proved by serial sections. Original magnification $\times 259$.

icular septae were found in 32 patients (Fig 6). So called "bridge-like channels" that often closely resemble Schlemm's canal were seen in serial sections of eight adult patients with open angle glaucomas (Fig 8). Heavy pigmentation of the whole trabecular meshwork was seen in 13 patients (two POAG, five PEX Gl, six Pigm Gl) and moderate pigmentation (Fig 8) in 19 patients (six POAG, 11 PEX Gl, two Pigm Gl), while in the remaining 68 patients pigmentation of the excised trabecular meshwork was very weak or even lacking. Out of the four patients from the Middle East two showed moderate and one heavy pigmentation of the meshwork, but in a 4 year old child from this region pigmentation was hardly visible.

\section{Discussion}

New modes of non-perforating antiglaucomatous surgery such as deep sclerectomy or viscocanalostomy aim to preserve the integrity of the trabecular meshwork in order to avoid overfiltration and irritation of the anterior chamber. In initial clinical studies satisfactory results have been reported even after longer follow ups. ${ }^{135}$ However, the key to success with these non-perforating techniques is a meticulous dissection of the deep scleral flap including removal of the outer wall of Schlemm's canal ("deroofing") without violating the fragile and loose anterior trabecular meshwork. This requires excellent knowledge of topographic variabilities and superb skill in lamellar microdissecting. In several studies the importance of individual surgical experience was stressed because intraoperative problems, such as inadvertent perforation of the trabeculo-descemetic membrane, were found to occur mainly in the surgeon's first 10 deep sclerectomies. $^{589}$ Viscocanalostomy which proved remarkably successful in a black population ${ }^{3}$ might be easier to perform in black than in white patients, as the sclera is usually thicker and the trabecular meshwork darker than in white patients. In our mainly white population the histology of the trabeculectomy specimens from the upper circumference of the eye revealed little or no pigmentation in nearly $70 \%$ of the eyes operated. Although the number of patients from the Middle East were relatively low, a marked tendency towards stronger pigmentation was already obvious for this ethnic group. Thus, the surgical dissection of the deep scleral flap in white people seems to be even more demanding, because in these patients an important intraoperative topographic landmark is missing. Another problem hindering intraoperative identification of Schlemm's canal can be the presence of bridge-like drainage channels running tangentially to Schlemm's canal which have been previously described by Rohen. ${ }^{10}$ When dissecting the deep scleral flap and entering a bridge-like channel such this, misidentification of Schlemm's canal can lead the surgeon to dissect the deep scleral flap too superficially. Although we found these kinds of tangential drainage vessels in less than $10 \%$ of cases, they could be a source of considerable intraoperative confusion where trabecular pigmentation is lacking. In adult glaucoma patients Schlemm's canal was always found despite the frequent occurrence of intracanalicular tissue bands, and the anterior-posterior diameter almost always allowed the introduction of the most commonly used instruments for viscocanalostomy. However, with very young patients, who usually lack pigmentation of the meshwork, the chances of failing to find Schlemm's canal increase, partly because of its small size. On the other hand, the risk of inadvertent perforation of the trabeculodescemetic membrane seems to be somewhat lower in younger glaucoma patients, confirming the clinical experience of Sanchez and co-workers, ${ }^{8}$ who, in a series of 234 deep sclerectomies, found a higher incidence of intraoperative perforation in the older patients. While the smaller dimensions of Schlemm's canal in children are well known, ${ }^{10}{ }^{11}$ a greater thickness of the anterior trabecular meshwork in younger glaucoma patients compared with older ones has not been reported before. There may be physiological reasons for this such as the embryological development of the drainage vessels emerging from the scleral side to the chamber angle region ${ }^{12}$ or the flattening of the trabecular architecture and fusing of beams with age. ${ }^{13}{ }^{14}$ However, no significant influence of the maximal recorded intraocular pressure on the trabecular thickness could be established in our study, although this cannot be categorically excluded owing to the considerable variability of our measurements. A critical point may be the fact that the so called "maximal recorded IOP" was not obtained in a uniform manner, but usually according to the patient's own description. Possibly, this value does not always reflect "the representative IOP" for each glaucoma patient. Finally, it is not unlikely that factors other than the maximum IOP rise are associated with the altered morphology of the trabecular meshwork in glaucoma patients and that each of them contributes to the morphological changes without reaching statistical significance in our limited and very heterogeneous patient selection.

Any quantitative study of trabeculectomy specimens is liable to suffer from preparation artefacts. ${ }^{15-17}$ Although surgical excision has 
been improved over the years and surgical artefacts haven been reduced as far as possible, neither accidental tissue distortion nor artificial enlargement of the tissue dimensions through the sectioning of a slightly tilted embedded tissue block can be completely ruled out. Another point of concern might be the fixation of the trabecular meshwork by immersion instead of the ideal technique of rapid high pressure fixation of the drainage pathways. The immersion fixation causes several important artefactual changes to the in vivo morphology of the human trabecular meshwork, including changes of the thickness of the meshwork and the cross sectional area of Schlemm's canal. ${ }^{15} 1819$ A sudden drop of the intraocular pressure during trabeculectomy may cause a collapse or narrowing of the trabecular architecture thus falsely indicating less thickness of the trabecular meshwork. However, regarding the usual technique of deep sclerectomy it has to be considered that most surgeons similarly induce a temporarily limited ocular hypotony (by a corneal incision) when dissecting the anterior part of the deep scleral flap in order to minimise the risk of inadvertent perforation by a direct or indirect trauma.

Another theoretical problem might be the segmental variability of the trabecular meshwork in the glaucomatous eye, because the excised trabeculectomy specimen only represents a very small portion of the whole drainage system. Fortunately, both the upper quadrants of the eye are the preferred areas for trabeculectomy as well as for non-perforating surgery so that this presents no topographic problem. Besides, a detailed previous study revealed that segmental variability is highest in the juxtacanalicular tissue and empty spaces, while most of the variables measured in the meshwork were relatively stable throughout the four quadrants, with a variability of approximately $20 \% .{ }^{20}$ Considering all these possible sources of error in quantitative analysis of trabeculectomy specimens, we were surprised that our data for the meridional diameter of Schlemm's canal and for the thickness of the trabecular meshwork was within the same range as the results of previous investigations. ${ }^{10111820-22}$ It was also found that statistical significance was reached for the age dependency of quantitative variables, confirming previous studies about progressive trabecular meshwork narrowing with age. ${ }^{10} 13$ However, it should be pointed out that within the group of adult patients with open angle glaucomas alone we found no evidence of age dependent changes in the dimensions of Schlemm's canal or the trabecular meshwork. This agrees with early studies of Rohen who stated that the size of Schlemm's canal becomes relatively stable after the age of 5 years. Judging by the findings of Lütjen and Rohen $^{11}$ and Nesterov ${ }^{21} 22$ the mean sagittal diameter of Schlemm's canal in our study is slightly smaller than in normal eyes. Buller and Johnson ${ }^{20}$ obtained a surprisingly similar length to ours for Schlemm's canal in glaucomatous eyes, while Gottanka et $a l^{23}$ found even shorter lengths, which correlated neither with age nor stage of disease. The smaller size of Schlemm's canal in a limited number of glaucoma patients with pathological outflow facility has previously been thought to reflect a reduced drainage capacity, ${ }^{24}$ although in our patient selection we found no correlation between maximum intraocular pressure or stage of the disease and the anterior-posterior diameter of Schlemm's canal. Qualitative assessment of the trabeculectomy specimens in our patient selection is also of importance, because the incidence of endothelial covering of the meshwork seems to be much higher than expected. The uveal meshwork was covered by an endothelial layer not only in secondary and infantile glaucomas, but also in a considerable number of patients with open angle glaucoma and a history of laser trabeculoplasty. Nonperforating surgery, such as viscocanalostomy, does not dissolve ab interno sealing of the meshwork and may therefore be unsuccessful in patients with secondary glaucoma and in glaucoma patients following laser trabeculoplasty. Although this problem is encountered in association with trabeculectomy ${ }^{25}{ }^{26}$ the theory of pressure reduction in such cases is even less logical for a non-perforating surgery than for a filtering procedure.

We conclude that intraoperative problems, such as inadvertent rupture of the trabeculodescemetic membrane or misidentification of Schlemm's canal, may not be related solely to the surgeon's experience, but could arise from the morphological variability of the aqueous drainage ways of glaucoma patients. ${ }^{27}{ }^{28}$ As already described in clinical studies, the risk of perforation is especially high in the region of the anterior trabecular meshwork and more pronounced in older patients than in younger ones. ${ }^{8}$ From a morphological point of view, the ultrasound biomicroscopic data obtained after deep sclerectomy ${ }^{29}$ which indicated a trabeculo-descemetic membrane of $130 \mu \mathrm{m}$ are in good accordance with the dimensions of the posterior meshwork, but suggest that the level of dissection was too superficial in the region of the anterior meshwork underlining the difficulty of achieving complete deroofing of Schlemm's canal. The relatively high incidence of endothelial covering of the drainage system raises the question of whether non-perforating antiglaucomatous procedures should be performed at all in secondary glaucomas or after laser trabeculoplasty.

\footnotetext{
1 Zimmerman T, Kooner K, Ford V, et al. Tra.beculectomy vs non-penetrating trabeculectomy: a retrospective study of two procedures in phakic patients with glaucoma. Ophthalmic Surg 1984;15:734-740.

2 Fjodorov SN, Ioffee DI, Ronkina TI. Deep sclerectomy: technique and mechanism of a new glaucomatous procedure. Glaucoma $1984 ; 6: 281-283$.

3 Stegmann R, Pienaar A, Miller D. Viscocanalostomy for open-angle glaucoma in black patients. F Cataract Refract Surg 1999;25:316-322.

4 Kozlov VI, Bagrov SN, Anisimova SY, et al. Nonpenetrating deep sclerectomy with collagen. IRTC eye microsurgery. Moscow: RSFSR Ministry of Public Health 1989;3:44-6.

5 Mermoud A, Schnyder CC, Sickenberg M, et al. Comparison of deep sclerectomy with collagen implant and trabeculectomy in open-angle glaucoma. $\mathcal{F}$ Cataract Refract Surg 1999;25:323-31.

6 Watson PG, Jakeman C, Ozturk M, et al. The complications of trabeculectomy (a 20 year follow-up). Eye 1990;4:425-38.
} 
7 Carassa RG, Bettin P, Fiori M, et al. Viscocanalostomy: a

pilot study. Eur f Ophthalmol 1998;8:57-61. Sanchez E, Schnyder CC, Mermoud A. Comparative result of deep sclerectomy transformed in trabeculectomy and those of standard trabeculectomy. Klin Monatsbl Augenheilkd 1997;210:261-4.

9 Welsh NH, DeLange J, Wasserman P, et al. The "deroofing" of Schlemm's canal in patients with open-angle glaucoma through placement of a collagen drainage device. Ophthalmic Surg Lasers 1998;29:216-26.

10 Rohen JW. New studies on the functional morphology of the trabecular meshwork and the outflow channels. Trans Ophthalmol Soc UK 1970;89:431-47.

11 Lütjen E, Rohen JW. Histometrische Untersuchungen über die Kammerwinkelregion des menschlichen Auges bei verschiedenen Altersstufen und Glaukomformen. Graefes Arch Clin Exp Ophthalmol 1968;176:1-12.

12 Hamanaka T, Bill A, Ichinohasama R, et al. Aspects of the development of Schlemm's canal. Exp Eye Res 1992;55: developr

13 Miyazaki M, Segawa K, Urakawa Y. Age-related changes in the trabecular meshwork of the normal human eye. $尹$ pn $\mathcal{F}$ Ophthalmol 1987;31:558-69.

14 McMenamin PG, Lee WR, Aitken DAN. Age-related changes in the human outflow apparatus. Ophthalmology 1986;93:194-209.

15 Lee WR. Doyne Lecture: the pathology of the outflow system in primary and secondary glaucoma. Eye 1995;9:1-23.

16 Rodrigues MM, Spaeth GL, Sivalingham E, et al.Value of trabeculectomy specimens in glaucoma. Ophthalmic Surg 1978;9:29-38.

17 Rodrigues MM, Spaeth GL, Sivalingham E. Histopathology of 150 trabeculectomy specimens in glaucoma. Trans Ophthalmol Soc UK 1976;96:245-55.

18 Ainsworth JR, Lee WR. Effects of age and rapid highpressure fixation on the morphology of Schlemm's canal. Invest Ophthalmol Vis Sci 1990;31:745-50.
19 Lee WR, Grierson I, McMenamin PG. The morphological response of the primate outflow system to changes in pressure and flow. laucoma research. Vol 2. New York: Schattauer, 1982:123-

20 Buller C, Johnson D. Segmental variability of the trabecular meshwork in normal and glaucomatous eyes. Invest Ophthalmol Vis Sci 1994;35:3841-51.

21 Nesterov AP, Batmanov YE. Study on morphology and function of the drainage area of the eye of man. Acta Ophthalmol 1972;50:337-349.

22 Nesterov AP, Hasanova NH, Batmanov YE. Schlemm's canal and scleral spur in normal and glaucomatous eyes. Acta Ophthalmol 1974;52:634-46.

23 Gottanka J, Johnson DH, Martus P, et al. Severity of optic nerve damage in eyes with POAG is correlated with changes in the trabecular meshwork. F Glaucoma 1997;6: 123-32.

24 Allingham RR, de Kater AW, Ethier CR. Schlemm's canal and primary open angle glaucoma:Correlation between Schlemm's canal dimensions and outflow facility. Exp Eye Res 1996;62:101-9.

25 Alexander RA, Grierson I, Church WH. The effect of argon aser trabeculoplasty upon the normal human trabecular meshwork. Graefes Arch Clin Exp Ophthalmol 1989;227:72-

26 Rodrigues MM, Spaeth GL, Donohoo P. Electron microscopy of argon laser therapy in phakic open-angle glaucoma. Ophthalmology 1982;89:198-210.

27 Khaw PT, Siriwardena D. "New" surgical treatments for glaucoma (Editorial). Br f Ophthalmol 1999;83:1-3.

28 Krieglstein GK. How new is new, and is it better? (Editorial). f Glaucoma 1999;8:279-80.

29 Chiou AGY, Mermoud A, Underdahl JP, et al. An ultrasound biomicroscopic study of eyes after deep sclerectomy with collagen implant. Ophthalmology 1998;105:74650 . 